\title{
On Mubayi's Conjecture and conditionally intersecting sets
}

\author{
Adam Mammoliti, * \\ a.mammoliti@unsw.edu.au \\ Thomas Britz* \\ britz@unsw.edu.au
}

\begin{abstract}
Mubayi's Conjecture states that if $\mathcal{F}$ is a family of $k$-sized subsets of $[n]=\{1, \ldots, n\}$ which, for $k \geq d \geq 3$ and $n \geq \frac{d k}{d-1}$, satisfies $A_{1} \cap \cdots \cap A_{d} \neq \emptyset$ whenever $\left|A_{1} \cup \cdots \cup A_{d}\right| \leq 2 k$ for all distinct sets $A_{1}, \ldots, A_{d} \in \mathcal{F}$, then $|\mathcal{F}| \leq\left(\begin{array}{c}n-1 \\ k-1\end{array}\right)$, with equality occurring only if $\mathcal{F}$ is the family of all $k$-sized subsets containing some fixed element. This paper proves that Mubayi's Conjecture is true for all families that are invariant with respect to shifting; indeed, these families satisfy a stronger version of Mubayi's Conjecture. Relevant to the conjecture, we prove a fundamental bijective duality between what we call $(i, j)$ unstable families and $(j, i)$-unstable families. Generalising previous intersecting conditions, we introduce the $(d, s, t)$-conditionally intersecting condition for families of sets and prove general results thereon. We prove fundamental theorems on two $(d, s)$-conditionally intersecting families that generalise previous intersecting families, and we pose an extension of a previous conjecture by Frankl and Füredi. Finally, we generalise a classical result by Erdős, Ko and Rado by proving tight upper bounds on the size of $(2, s)$-conditionally intersecting families $\mathcal{F} \subseteq 2^{[n]}$ and by characterising the families that attain these bounds. We extend this theorem for sufficiently large $n$ to families $\mathcal{F} \subseteq 2^{[n]}$ whose members have at most a fixed size $u$.
\end{abstract}

Keywords: Extremal set theory, intersecting sets, the Erdős-Ko-Rado Theorem, Mubayi's Conjecture, unstable MSC subject classifications: 05D05, 05C35, 05C65

\section{Introduction}

Let $\mathcal{F} \subseteq\left(\begin{array}{c}{[n]} \\ k\end{array}\right)$ be a family of $k$-sized subsets of $[n]:=\{1, \ldots, n\}$. The celebrated Erdös-KoRado Theorem [5] states that if $n \geq 2 k$ and $A \cap B \neq \emptyset$ for all sets $A, B \in \mathcal{F}$, then $|\mathcal{F}| \leq\left(\begin{array}{l}n-1 \\ k-1\end{array}\right)$, with equality when $n>2 k$ occurring precisely when $\mathcal{F}$ is a star, i.e., when $\mathcal{F}$ is the family of all $k$-sized sets that contain a fixed element of $[n]$. As Frankl [6] proved, this theorem still holds when the intersection condition $A \cap B \neq \emptyset$ is replaced by the more general condition that any $d$ sets of $\mathcal{F}$ have nonempty intersection, under the assumption that $n \geq d k /(d-1)$ :

${ }^{*}$ School of Mathematics and Statistics UNSW Sydney NSW 2052, Australia 
Theorem 1. If $\mathcal{F} \subseteq\left(\begin{array}{c}{[n]} \\ k\end{array}\right)$ is a family of $k$-subsets of $[n]$ where $n \geq \frac{d k}{d-1}$ and $k \geq d \geq 3$ so that, for each $d$ sets $A_{1}, \ldots, A_{d} \in \mathcal{F}$,

$$
A_{1} \cap \cdots \cap A_{d} \neq \emptyset
$$

then

$$
|\mathcal{F}| \leq\left(\begin{array}{l}
n-1 \\
k-1
\end{array}\right)
$$

Furthermore, equality holds if and only if $\mathcal{F}$ is a star.

According to Frankl and Füredi [10], Katona sought to extend the $d=3$ case of Frankl's result, by relaxing the intersection condition (10) to be required only when any $d=3$ distinct sets of $\mathcal{F}$ have union containing at most $s$ elements for some $s \leq 3 k$. Frankl and Füredi [10] proved that this was possible when $2 k \leq s \leq 3 k$ and $n$ is sufficiently large. Mubayi [16] completed this work by proving that the $d=3$ case of Theorem 1 could indeed be extended whenever $n \geq 3 k / 2$. This led Mubayi [16] to conjecture a further extension to all values of $d$, as follows:

Conjecture 2. [16] If $\mathcal{F} \subseteq\left(\begin{array}{c}{[n]} \\ k\end{array}\right)$ is a family of $k$-subsets of $[n]$ where $n \geq \frac{d k}{d-1}$ and $k \geq d \geq 3$ so that, for all distinct sets $A_{1}, \ldots, A_{d} \in \mathcal{F}$,

$$
A_{1} \cap \cdots \cap A_{d} \neq \emptyset \quad \text { whenever } \quad\left|A_{1} \cup \cdots \cup A_{d}\right| \leq 2 k,
$$

then

$$
|\mathcal{F}| \leq\left(\begin{array}{l}
n-1 \\
k-1
\end{array}\right)
$$

Furthermore, equality holds if and only if $\mathcal{F}$ is a star.

As described above, Mubayi [16] proved the conjecture for $d=3$, and Mubayi [17] proved the conjecture for $d=4$ when $n$ is sufficiently large. Füredi and Özkahya [1] and Mubayi and Ramadurai [18] independently improved this result by proving the conjecture for sufficiently large $n$, thus generalising the above-mentioned result by Frankl and Füredi [10. Chen et al. [4] proved Mubayi's Conjecture for $d=k$ and Füredi and Özkahya [11] proved that Mubayi's Conjecture even holds when $d=k+1$. However, Mubayi [17] provided a counterexample that showed that the conjecture could not be extended to values of $d$ greater than or equal to $2^{k}$. During the publication of this article, Lifshitz [15] also proved that Mubayi's Conjecture holds when $0<\zeta n \leq k \leq \frac{d-1}{d} n$ and $n$ is sufficiently larger than $\zeta$ and $d$, and that, under these conditions, the upper bound in Condition (2) can indeed be relaxed to $\left(\frac{d}{d-1}+\zeta\right) k$.

The first main result of the present paper, Theorem 5 in Section [2, is a new partial verification of Mubayi's Conjecture. Namely, we prove that Mubayi's Conjecture holds for stable families $\mathcal{F} \subseteq\left(\begin{array}{c}{[n]} \\ k\end{array}\right)$ of $k$-sets; these are the families that are invariant with respect to the shifting operation. Indeed, it turns out that Mubayi's Conjecture holds for such families even when the upper bound in Condition (2) of the conjecture is relaxed.

Thus, to prove or disprove Mubayi's Conjecture, it is sufficient to consider the conjecture with respect to families that are not stable under shifting. In Section 3, we prove general 
properties and characterisations of families that are unstable and, more subtly, that are $(i, j)$ unstable. The main result of that section is Theorem 12 which describes how each $(i, j)-$ unstable family is related by an explicit and fundamental involution to a unique $(j, i)$-unstable family.

These notions of stability refer to the central concept of this paper, namely the $(d, s, t)$ conditionally intersecting condition for families of sets, introduced in Section 3. For $t=1$, this becomes the $(d, s)$-conditionally intersecting condition which, in turn for $s=2 k$, is the intersecting condition (2). More generally, the $(d, s, t)$-conditionally intersecting condition naturally generalises many previous intersecting conditions in the literature [2, 5, 6, 10, 9, 18]; those are here given a useful common framework.

In Section 4, we pose a conjecture that sharpens Mubayi's Conjecture by considering the difference between the two conditions in Frankl's Theorem and Mubayi's Conjecture. In particular, we conjecture on the size and extremal structures of families $\mathcal{F} \in\left(\begin{array}{c}{[n]} \\ k\end{array}\right)$ that are $(d, 2 k)$-conditionally intersecting but which are not intersecting; see Conjecture 13. We show by example that this conjecture cannot be extended to small values of $n$ and we also show that there is circumstantial reason to believe that Conjecture13 might be true: Proposition 14 shows that a similar claim is true for $(3,4)$-conditionally intersecting families.

The families $\mathcal{F} \in\left(\begin{array}{c}{[n]} \\ k\end{array}\right)$ that are $(d, s)$-conditionally intersecting for some $s<2 k$ include several intersection families in the literature [1, 2, 5, 10]. In Section [5, we therefore define two $(d, s)$-conditionally intersecting families that generalise these previous families. We prove a fundamental theorem (Theorem 20) on these new families and thus also on previous families, and we pose Conjecture 15 which extends a previous conjecture by Frankl and Füredi [10] on $(3,2 k-1)$-conditionally intersecting families.

The final Sections [ 6 and 7 are motivated by the classical result by Erdös et al. [5] that an intersecting family $\mathcal{F} \subseteq 2^{[n]}$ can have size at most $2^{n-1}$ and that this bound is met by star families. In Section [6, we generalise this theorem by proving tight upper bounds on the size of $(2, s)$-conditionally intersecting families $\mathcal{F} \subseteq 2^{[n]}$ and by characterising the families that attain these bounds; see Theorem 23. We extend these results further in Section 7 , for certain parameters as well as for sufficiently large families with respect to $(2, s)$-conditionally intersecting families $\mathcal{F} \subseteq 2^{[n]}$ whose members have at most a fixed number $u$ members; see Theorems 25] and 27. Finally, we invite the reader to ponder the question of whether these final results can be merged into a complete description of $(2, s)$-conditionally intersecting families $\mathcal{F} \subseteq 2^{[n]}$ and their extremal sizes and structures.

\section{Mubayi's Conjecture for stable families}

Initially used to prove the Erdős-Ko-Rado Theorem [5] and surveyed in [9, 7], the $(i, j)$-shift $S_{i j}$ on each family $\mathcal{F} \subseteq 2^{[n]}$ is the function defined by

$$
S_{i j}(\mathcal{F}):=\left\{S_{i j}(A): A \in \mathcal{F}\right\},
$$

where

$$
S_{i j}(A):= \begin{cases}A^{\prime}=(A-\{j\}) \cup\{i\} & \text { if } j \in A, i \notin A, A^{\prime} \notin \mathcal{F} \\ A & \text { otherwise. }\end{cases}
$$


A few properties of the shift $S_{i j}$ are given below.

Proposition 3. If $\mathcal{F} \subseteq 2^{[n]}$ and $i, j \in[n]$, then

(i) $\left|S_{i j}(A)\right|=|A|$ for all $A \in \mathcal{F}$;

(ii) $\left|S_{i j}(\mathcal{F})\right|=|\mathcal{F}|$;

(iii) for any $\mathcal{G} \subseteq \mathcal{F},\left|\bigcup_{A \in \mathcal{G}} A\right|-1 \leq\left|\bigcup_{A \in \mathcal{G}} S_{i j}(A)\right| \leq\left|\bigcup_{A \in \mathcal{G}} A\right|+1$ and

$$
\left|\bigcap_{A \in \mathcal{G}} A\right| \leq\left|\bigcap_{A \in \mathcal{G}} S_{i j}(A)\right| \leq\left|\bigcap_{A \in \mathcal{G}} A\right|+1
$$

Proof. (i) and (ii) follow from definitions (see also [7]). To prove (iii), note that the shift operation cannot increase or decrease the size of a union or intersection of sets $A \in \mathcal{G}$ by more than one. Suppose that $\left|\bigcap_{A \in \mathcal{G}} S_{i j}(A)\right|=\left|\bigcap_{A \in \mathcal{G}} A\right|-1$. Then the shift $S_{i j}$ has removed $j$ from the intersection and yet did not add $i$, so $i$ must already be contained in $\bigcap_{A \in \mathcal{G}} A$ and thus in each member $A \in \mathcal{G}$, a contradiction.

If $S_{i j}(\mathcal{F})=\mathcal{F}$ whenever $1 \leq i<j \leq n$, then $\mathcal{F}$ is stable or shifted. When $i<j$, the set $S_{i j}(A)$ either equals $A$ or replaces an element in $A$ by an element of smaller value, so each member $A$ of a stable family $\mathcal{F}$ must satisfy $S_{i j}(A)=A$. Applying sufficiently many shifts $S_{i_{\ell} j_{\ell}}$ with $i_{\ell}<j_{\ell}$ to $\mathcal{F}$ will yield a (non-unique) stable family $\mathcal{F}^{\prime}$; see [7].

Example 4. The family $\mathcal{F}:=\{\{1,2\},\{1,3\}\} \subseteq 2^{[3]}$ is stable. More generally, each star $\mathcal{F} \subseteq\left(\begin{array}{c}n] \\ k\end{array}\right)$ whose members each contain the element 1 are stable.

The main result of the paper is Theorem 5 below which states that Mubayi's Conjecture holds for stable families $\mathcal{F}$ of $k$-sets even when Condition (2) of the conjecture is relaxed.

Theorem 5. If $\mathcal{F} \subseteq\left(\begin{array}{c}{[n]} \\ k\end{array}\right)$ is a stable family of $k$-subsets of $[n]$ where $n \geq \frac{d k}{d-1}$ and $k \geq d \geq 3$ so that, for all distinct sets $A_{1}, \ldots, A_{d} \in \mathcal{F}$,

$$
A_{1} \cap \cdots \cap A_{d} \neq \emptyset \text { whenever }\left|A_{1} \cup \cdots \cup A_{d}\right| \leq 2 k-(d-2) \text {, }
$$

then

$$
|\mathcal{F}| \leq\left(\begin{array}{l}
n-1 \\
k-1
\end{array}\right)
$$

Furthermore, equality holds if and only if $\mathcal{F}$ is a star.

Proof. Suppose that $\mathcal{F}$ is a family as given in the theorem.

If $n \leq 2 k-(d-2)$, then $\left|A_{1} \cup \cdots \cup A_{d}\right| \leq 2 k-(d-2)$ is always true, so Condition (3) simply requires that any $d$ members of $\mathcal{F}$ intersect, and the theorem follows from Theorem 1 .

Suppose now that $n>2 k-(d-2)$ and assume inductively that the theorem is true for all integers $m=2 k-(d-2), \ldots, n-1$ and $d$ and $k$ for which $k \geq d \geq 3$ and $m \geq \frac{d k}{d-1}$. Define

$$
\mathcal{F}(\bar{n}):=\{A: n \notin A \in \mathcal{F}\} \quad \text { and } \quad \mathcal{F}(n):=\{A \backslash\{n\}: n \in A \in \mathcal{F}\} .
$$


Since $\mathcal{F}(\bar{n}) \subseteq \mathcal{F}$ and since $\mathcal{F}$ satisfies Condition (3), the family $\mathcal{F}(\bar{n})$ must satisfy Condition (3). The family $\mathcal{F}(\bar{n})$ is clearly invariant under any shift of the form $S_{i n}$. As $\mathcal{F}$ is stable, it follows that $A^{\prime}=(A-\{j\}) \cup\{i\} \in \mathcal{F}$ for any $i<j<n$ and $A \in \mathcal{F}(\bar{n})$ such that $j \in A$ and $i \notin A$. Furthermore, $A^{\prime} \in \mathcal{F}(\bar{n})$ since $n \notin A^{\prime}$. Thus, $\mathcal{F}(\bar{n})$ is stable and, by assumption, $|\mathcal{F}(\bar{n})| \leq\left(\begin{array}{l}n-2 \\ k-1\end{array}\right)$.

Now consider $\mathcal{F}(n)$. Let $S_{i j}$ be a shift with $i<j<n$, let $A \in \mathcal{F}(n)$, and set $B=A \cup\{n\}$. Since $\mathcal{F}$ is stable, it follows that $S_{i j}(A)=S_{i j}(B)-\{n\}=B-\{n\}=A$, so $\mathcal{F}(n)$ is stable. Assume that $\mathcal{F}(n)$ does not satisfy Condition (3) for the value $d-1$. That is, assume that $A_{1} \cap \cdots \cap A_{d-1}=\emptyset$ for some members $A_{1}, \ldots, A_{d-1} \in \mathcal{F}(n)$ with

$$
\left|A_{1} \cup \cdots \cup A_{d-1}\right| \leq 2(k-1)-((d-1)-2) .
$$

Set $F_{j}:=A_{j} \cup\{n\}$ for each $j=1, \ldots, d-1$. By definition, $F_{1} \cap \cdots \cap F_{d-1}=\{n\}$ and $F_{j} \in \mathcal{F}$. Choose $i \in A_{1} \cup \cdots \cup A_{d-1}$ and $A_{\ell}$ such that $i \notin A_{\ell}$, and define the set $F_{\ell}^{\prime}:=\left(F_{\ell}-\{n\}\right) \cup\{i\}$. Since $\mathcal{F}$ is stable and $i \notin F_{\ell}$ and $n \in F_{\ell}$, it follows that $F_{\ell}^{\prime} \in \mathcal{F}$. Then by (4),

$$
\left|F_{1} \cup F_{2} \cup \cdots \cup F_{d-1} \cup F_{\ell}^{\prime}\right| \leq 2(k-1)-((d-1)-2)+1=2 k-(d-2)
$$

but

$$
F_{1} \cap \cdots \cap F_{d-1} \cap F_{\ell}^{\prime}=\emptyset,
$$

a contradiction. Hence, $\mathcal{F}(n)$ is stable and satisfies Condition (3) for the value $d-1$. If $d>3$, then $k-1 \geq d-1 \geq 3$ and, as initially supposed,

$$
n-1 \geq 2 k-(d-2)=\frac{d-1}{d-2}(k-1)+\frac{(d-3)(k-d+2)+1}{d-2}>\frac{d-1}{d-2}(k-1)
$$

so by the induction assumption, $|\mathcal{F}(n)| \leq\left(\begin{array}{c}n-2 \\ k-2\end{array}\right)$. If $d=3$, then $n-1 \geq 2 k-(d-2)=$ $2 k-1>2(k-1)$, and since Condition (3) for $\mathcal{F}(n)$ reduces to the condition of the Erdös-KoRado Theorem for $d-1$, it follows from that theorem that $|\mathcal{F}(n)| \leq\left(\begin{array}{l}n-2 \\ k-2\end{array}\right)$. Hence,

$$
|\mathcal{F}|=|\mathcal{F}(\bar{n})|+|\mathcal{F}(n)| \leq\left(\begin{array}{l}
n-2 \\
k-1
\end{array}\right)+\left(\begin{array}{l}
n-2 \\
k-2
\end{array}\right)=\left(\begin{array}{l}
n-1 \\
k-1
\end{array}\right) .
$$

If $\mathcal{F}$ is a star, then equality is trivially attained above. Conversely, suppose that $|\mathcal{F}|=$ $\left(\begin{array}{l}n-1 \\ k-1\end{array}\right)$. Then, by the inequality above, $|\mathcal{F}(\bar{n})|=\left(\begin{array}{l}n-2 \\ k-1\end{array}\right)$ and $|\mathcal{F}(n)|=\left(\begin{array}{l}n-2 \\ k-2\end{array}\right)$. By the induction assumption, $\mathcal{F}(\bar{n})$ forms a star and if $d>3$, then $\mathcal{F}(n)$ also forms a star. By the Erdös-KoRado Theorem, $\mathcal{F}(n)$ also forms a star when $d=3$. Let $j$ denote the common element of the members of $\mathcal{F}(n)$, and assume that $j \neq 1$. Since $n-1 \geq k$, there exists $A \in \mathcal{F}(n)$ such that $1 \notin A$. Thus, $S_{1 j}(A)=(A-\{j\}) \cup\{1\} \in \mathcal{F}(n)$ as $\mathcal{F}(n)$ is stable. But $j \notin S_{1 j}(A)$, a contradiction, so $j=1$. Similarly, the common element of the members of $\mathcal{F}(\bar{n})$ must be 1 . Hence, $\mathcal{F}$ is the star $\left\{A: 1 \in A \in\left(\begin{array}{c}{[n]} \\ k\end{array}\right)\right\}$. Induction concludes the proof.

\section{A closer look at intersecting conditions}

Theorem 5 verifies Mubayi's Conjecture for stable families and indeed provides a stronger result for these families. The following observation is thus worth highlighting. 
Corollary 6. To prove or disprove Mubayi's Conjecture, it is sufficient to consider the conjecture with respect to families that are not stable under shifting.

Given this observation, it is natural and perhaps important to investigate the general properties of families that are not stable; that is the purpose of the present section.

A family of subsets $\mathcal{F} \subseteq 2^{[n]}$ is $(d, s, t)$-conditionally intersecting if, for all distinct sets $A_{1}, \ldots, A_{d} \in \mathcal{F}$

$$
\left|A_{1} \cap \cdots \cap A_{d}\right| \geq t \quad \text { whenever } \quad\left|A_{1} \cup \cdots \cup A_{d}\right| \leq s .
$$

If $\mathcal{F}$ is $(d, s, 1)$-conditionally intersecting, then $\mathcal{F}$ is $(d, s)$-conditionally intersecting. Thus, the Erdős-Ko-Rado Theorem addresses families $\mathcal{F}$ that are $(2, n)$-conditionally intersecting. Similarly, families $\mathcal{F}$ satisfying Conditions (11), (2) and (3) are, respectively, $(d, n)$-conditionally intersecting, $(d, 2 k)$-conditionally intersecting, and $(d, 2 k-(d-3))$-conditionally intersecting. A family $\mathcal{F}$ is $d$-wise $t$-intersecting if it is $(d, n, t)$-conditionally intersecting; that is, if

$$
\left|A_{1} \cap \cdots \cap A_{d}\right| \geq t \quad \text { whenever } \quad A_{1}, \ldots, A_{d} \in \mathcal{F} .
$$

Families of $d$ sets $A_{1}, \ldots, A_{d}$ which do not $(d, 2 k)$-conditionally intersect have been referred to in the literature as $d$-clusters; see [4, 11, 14, 18, 19].

The following proposition lists some observations regarding $(d, s, t)$-conditionally intersecting families and shifting.

Proposition 7. If $\mathcal{F} \subseteq\left(\begin{array}{c}{[n]} \\ k\end{array}\right)$ and $i, j \in[n]$, then

(i) if $\mathcal{F}$ is $(d, s, t)$-conditionally intersecting, then $\mathcal{F}$ is $(d, s-1, t)$-conditionally intersecting;

(ii) if $\mathcal{F}$ is $(d, s, t)$-conditionally intersecting and $s \geq d k$, then $\mathcal{F}$ is $d$-wise t-intersecting;

(iii) if $\mathcal{F}$ is d-wise t-intersecting, then so is the shift $S_{i j}(\mathcal{F})$.

Proof. (i) and (ii) follow easily from definitions. Proposition 3 (iii) implies (iii) (see also [7]).

Shifting does not always preserve the $(d, s, t)$-conditionally intersecting property. The following proposition presents cases in which shifting does preserve the $(d, s, t)$-conditionally intersecting property.

Proposition 8. If $A_{1}, \ldots, A_{d} \in 2^{[n]}$ are distinct and either

$$
\left|A_{1} \cap \cdots \cap A_{d}\right| \geq t \quad \text { or } \quad\left|A_{1} \cup \cdots \cup A_{d}\right| \geq s+2,
$$

then $\left\{S_{i j}\left(A_{1}\right), \ldots, S_{i j}\left(A_{d}\right)\right\}$ is $(d, s, t)$-conditionally intersecting.

Proof. If $\left|A_{1} \cap \cdots \cap A_{d}\right| \geq t$, then $\left\{A_{1}, \ldots, A_{d}\right\}$ is $d$-wise $t$-intersecting, so by Proposition 7 (iii), the family $\left\{S_{i j}\left(A_{1}\right), \ldots, S_{i j}\left(A_{d}\right)\right\}$ is also $d$-wise $t$-intersecting and thus $(d, s, t)$-conditionally intersecting. Now suppose that $\left|A_{1} \cup \cdots \cup A_{d}\right| \geq s+2$. By Proposition 3 (iii), $\mid S_{i j}\left(A_{1}\right) \cup \cdots \cup$ $S_{i j}\left(A_{d}\right) \mid \geq s+1$, so $\left\{S_{i j}\left(A_{1}\right), \ldots, S_{i j}\left(A_{d}\right)\right\}$ is trivially $(d, s, t)$-conditionally intersecting. 
Thus by this proposition, if $A_{1}, \ldots, A_{d} \in 2^{[n]}$ are distinct and the family $\left\{S_{i j}\left(A_{1}\right), \ldots, S_{i j}\left(A_{d}\right)\right\}$ is not $(d, s, t)$-conditionally intersecting, then

$$
\left|A_{1} \cap \cdots \cap A_{d}\right| \leq t-1 \quad \text { and } \quad\left|A_{1} \cup \cdots \cup A_{d}\right| \leq s+1 .
$$

Hence in order to characterise when the $(d, s, t)$-conditionally intersecting condition is not preserved under shifting, we must consider families $\mathcal{F} \in\left(\begin{array}{c}{[n]} \\ k\end{array}\right)$ that are $(d, s, t)$-conditionally intersecting while $S_{i j}(\mathcal{F})$ is not, for some $i, j$.

A family $\mathcal{F} \subseteq 2^{[n]}$ with this property is $(i, j)$-unstable and must contain a subfamily $\left\{A_{1}, \ldots, A_{d}\right\} \subseteq \mathcal{F}$ that is also $(i, j)$-unstable and thus satisfies

$$
\left|A_{1} \cap \cdots \cap A_{d}\right| \geq t \quad \text { or } \quad\left|A_{1} \cup \cdots \cup A_{d}\right| \geq s+1
$$

as well as the inequalities (6) and

$$
\left|S_{i j}\left(A_{1}\right) \cap \cdots \cap S_{i j}\left(A_{d}\right)\right| \leq t-1 \quad \text { and } \quad\left|S_{i j}\left(A_{1}\right) \cup \cdots \cup S_{i j}\left(A_{d}\right)\right| \leq s .
$$

Thus by Proposition [3, $(i, j)$-unstable families may be characterised as follows.

Proposition 9. A family of d distinct sets $\mathcal{A}=\left\{A_{1}, \ldots, A_{d}\right\} \subseteq \mathcal{F} \subseteq 2^{[n]}$ is $(i, j)$-unstable if and only if

$$
\begin{aligned}
\left|A_{1} \cap \cdots \cap A_{d}\right| & \leq\left|S_{i j}\left(A_{1}\right) \cap \cdots \cap S_{i j}\left(A_{d}\right)\right| \quad \leq t-1 \quad \text { and } \\
\left|A_{1} \cup \cdots \cup A_{d}\right| & =\left|S_{i j}\left(A_{1}\right) \cup \cdots \cup S_{i j}\left(A_{d}\right)\right|+1=s+1 .
\end{aligned}
$$

Proof. If $\mathcal{A}$ is $(i, j)$-unstable, then (9) follows from (6), (7) and (8), using Proposition 3 , Conversely, if (9) is true, then $\left|A_{1} \cup \cdots \cup A_{d}\right|>s$, so $\left\{A_{1}, \ldots, A_{d}\right\}$ is $(d, s, t)$-conditionally intersecting - and, in contrast, $\left\{S_{i j}\left(A_{1}\right), \ldots, S_{i j}\left(A_{d}\right)\right\}$ is not: $\left|S_{i j}\left(A_{1}\right) \cap \cdots \cap S_{i j}\left(A_{d}\right)\right|<t$ and $\left|S_{i j}\left(A_{1}\right) \cup \cdots \cup S_{i j}\left(A_{d}\right)\right| \leq s$.

Example 10. The family $\mathcal{F}=\{\{1,3\},\{2,4\},\{3,5\}\}$ is $(3,4,1)$-conditionally intersecting and is $(1,2)$-unstable since the shift $S_{12}(\mathcal{F})=\{\{1,3\},\{1,4\},\{3,5\}\}$ is not $(3,4,1)$-conditionally intersecting.

The following lemma describes an important property of $(i, j)$-unstable subfamilies $\mathcal{A}$ of a $(d, s, t)$-conditionally intersecting family $\mathcal{F}$ that contain $d$ elements. In particular, the sets in $\mathcal{A}$ containing $j$ but not $i$ can be shifted non-trivially.

Lemma 11. If $\mathcal{A}=\left\{A_{1}, \ldots, A_{d}\right\} \subseteq \mathcal{F} \subseteq 2^{[n]}$ is $(i, j)$-unstable, then each set $A_{\ell}$ is contained in one of

$$
\begin{aligned}
& \mathcal{A}_{\overline{i j}}=\left\{A \in \mathcal{A}: j \in A, i \notin A, A^{\prime}=(A-\{j\}) \cup\{i\} \notin \mathcal{F}\right\}=\left\{A \in \mathcal{A}: S_{i j}(A) \neq A\right\} ; \\
& \mathcal{A}_{i \bar{j}}=\{A \in \mathcal{A}: j \notin A, i \in A\} ; \\
& \mathcal{A}_{\overline{i j}}=\{A \in \mathcal{A}: j \notin A, i \notin A\} .
\end{aligned}
$$

Furthermore, $\mathcal{A}_{\bar{i} j}$ and $\mathcal{A}_{i \bar{j}}$ are nonempty, and if $\left|A_{1} \cap \cdots \cap A_{d}\right|=t-1$, then $\mathcal{A}_{\bar{i} \bar{j}}$ is also nonempty. 
Proof. By Condition (9), $i, j \in A_{1} \cup \cdots \cup A_{d}, j \notin S_{i j}\left(A_{1}\right) \cup \cdots \cup S_{i j}\left(A_{d}\right)$, and $i \notin A_{1} \cap \cdots \cap A_{d}$. If $j \in A_{\ell}$, then $S_{i j}\left(A_{\ell}\right) \neq A_{\ell}$, so $i \notin A_{\ell}$ and $A_{\ell}^{\prime}=\left(A_{\ell}-\{j\}\right) \cup\{i\} \notin \mathcal{F}$. Hence, any set containing $j$ is contained in $\mathcal{A}_{\bar{i} j}$ and as $j \in A_{1} \cup \cdots \cup A_{d}, \mathcal{A}_{\bar{i} j}$ is nonempty. As each set containing $j$ does not contain $i$, each of the sets $A_{\ell}$ containing $i$ must be elements of $\mathcal{A}_{i \bar{j}}$. Also, $\mathcal{A}_{i \bar{j}}$ is non-empty since $i \in A_{1} \cup \cdots \cup A_{d}$. If $\left|A_{1} \cap \cdots \cap A_{d}\right|=t-1$, then $i \notin A_{1} \cap \cdots \cap A_{d}=S_{i j}\left(A_{1}\right) \cap \cdots \cap S_{i j}\left(A_{d}\right)$, so there exists $A_{\ell}$ such that $i \notin S_{i j}\left(A_{\ell}\right)$, which means $i, j \notin A_{\ell}$ and so the set $\mathcal{A}_{\overline{i j}}$ must be non-empty.

The main result of this section, below, shows how $(i, j)$-instability and $(j, i)$-instability form dual symmetries that are connected by simple bijections. In particular, swapping the sets in $A \in \mathcal{A}_{i \bar{j}}$ by $(A-\{i\}) \cup\{j\}$ whenever $A$ is fixed by $S_{j i}$ defines a bijection between $(i, j)$-unstable families $\mathcal{A}$ and $(j, i)$-unstable families $\mathcal{B}$.

Theorem 12. Suppose that $\mathcal{A}=\left\{A_{1}, \ldots, A_{d}\right\} \subseteq \mathcal{F} \subseteq 2^{[n]}$ is $(i, j)$-unstable. Let $\mathcal{A}_{\overline{i j}}$, $\mathcal{A}_{i \bar{j}}$ and $\mathcal{A}_{\overline{i j}}$ be defined as in Lemma 11, suppose that $\mathcal{A}_{\overline{i j}} \neq \emptyset$, and define

$$
\mathcal{G}=\left\{A \in \mathcal{A}_{i \bar{j}}: S_{j i}(A)=A\right\} \quad \text { and } \quad \mathcal{G}^{\prime}=\{(A-\{i\}) \cup\{j\}: A \in \mathcal{G}\} .
$$

Then $\mathcal{G} \neq \mathcal{A}_{i \bar{j}}$, and

$$
\mathcal{B}=\mathcal{A}_{\overline{i j}} \cup \mathcal{A}_{\bar{i} \bar{j}} \cup\left(\mathcal{A}_{i \bar{j}}-\mathcal{G}\right) \cup \mathcal{G}^{\prime}
$$

is a $(j, i)$-unstable family of $d$ sets $\left\{B_{1}, \ldots, B_{d}\right\} \subseteq \mathcal{F}$.

Conversely, define families $\mathcal{B}_{\bar{j} i}, \mathcal{B}_{\bar{j} \bar{i}}$ and $\mathcal{B}_{\bar{i} \bar{j}}$ analogously for $\mathcal{B}$ as in Lemma [1], Then

$$
\mathcal{A}=\mathcal{B}_{\overline{j i}} \cup \mathcal{B}_{\bar{i} \bar{j}} \cup\left(\mathcal{B}_{j \bar{i}}-\mathcal{H}\right) \cup \mathcal{H}^{\prime}
$$

where

$$
\mathcal{H}=\left\{B \in \mathcal{B}_{j \bar{i}}: S_{i j}(B)=B\right\} \quad \text { and } \quad \mathcal{H}^{\prime}=\{(B-\{j\}) \cup\{i\}: B \in \mathcal{H}\} .
$$

Proof. To prove that $\mathcal{B} \subseteq \mathcal{F}$, note that $\mathcal{A}_{\bar{i} j}, \mathcal{A}_{\bar{i} \bar{j}}, \mathcal{A}_{i \bar{j}} \subseteq \mathcal{A} \subseteq \mathcal{F}$ and that if $B=(A-\{i\}) \cup\{j\} \in$ $\mathcal{G}^{\prime}$ where $A \in \mathcal{G}$, then $B \in \mathcal{F}$ by definition of $S_{j i}$ since $S_{j i}(A)=A$ and $A \in \mathcal{A}_{i \bar{j}}$.

Next, note that

$$
\left|\left(\mathcal{A}_{i \bar{j}}-\mathcal{G}\right) \cup \mathcal{G}^{\prime}\right|=\left|\left\{A \in \mathcal{A}_{i \bar{j}}: S_{j i}(A) \neq A\right\}\right|+\left|\left\{A \in \mathcal{A}_{i \bar{j}}: S_{j i}(A)=A\right\}\right|=\left|\mathcal{A}_{i \bar{j}}\right|,
$$

so by Lemma 11 .

$$
|\mathcal{B}|=\left|\mathcal{A}_{\overline{i j}}\right|+\left|\mathcal{A}_{\overline{i j}}\right|+\left|\left(\mathcal{A}_{i \bar{j}}-\mathcal{G}\right) \cup \mathcal{G}^{\prime}\right|=\left|\mathcal{A}_{\overline{i j}}\right|+\left|\mathcal{A}_{\bar{i} \bar{j}}\right|+\left|\mathcal{A}_{i \bar{j}}\right|=|\mathcal{A}|=d .
$$

Let us now prove that $\mathcal{B}$ is $(j, i)$-unstable. As $\mathcal{B} \subseteq \mathcal{F}, \mathcal{B}$ is $(d, s, t)$-conditionally intersecting. Since $\mathcal{A}_{\bar{i} \bar{j}} \neq \emptyset$ and since the sets in $\mathcal{B}$ are equal to those in $\mathcal{A}$ or obtained from such by replacing $j$ with $i$, it follows that $i, j \notin B_{1} \cap \cdots \cap B_{d}$. Hence by Proposition 9 , $\left|B_{1} \cap \cdots \cap B_{d}\right|=$ $\left|A_{1} \cap \cdots \cap A_{d}\right| \leq t-1$. Similarly since $\mathcal{A}_{\overline{i j}} \subseteq \mathcal{B}$ is non-empty and unchanged by $S_{j i}$, it follows that $i, j \notin S_{j i}\left(B_{1}\right) \cap \cdots \cap S_{j i}\left(B_{d}\right)$, so $\left|S_{j i}\left(B_{1}\right) \cap \cdots \cap S_{j i}\left(B_{d}\right)\right|=\left|B_{1} \cap \cdots \cap B_{d}\right| \leq t-1$.

By Lemma 11, $\mathcal{A}_{i j}$ is nonempty, so $j$ is contained in $A_{1} \cup \cdots \cup A_{d}, B_{1} \cup \cdots \cup B_{d}$ and $S_{j i}\left(B_{1}\right) \cup \cdots \cup S_{j i}\left(B_{d}\right)$. Thus, these unions differ only in whether they contain $i$ or not. By definition of $\mathcal{G}, S_{j i}(B) \neq B$ for every $B \in \mathcal{A}_{i \bar{j}}-\mathcal{G}$. Thus, $i \notin S_{j i}\left(B_{1}\right) \cup \cdots \cup S_{j i}\left(B_{d}\right)$ since 
every set in $\mathcal{B}$ containing $i$ is in $A_{i \bar{j}}-\mathcal{G}$. By Lemma 11, $\mathcal{A}_{i \bar{j}}$ is nonempty so $i \in A_{1} \cup \cdots \cup A_{d}$, and by Proposition 9, $\left|A_{1} \cup \cdots \cup A_{d}\right|=s+1$. Hence,

$$
s=\left|S_{j i}\left(B_{1}\right) \cup \cdots \cup S_{j i}\left(B_{d}\right)\right| \leq\left|B_{1} \cup \cdots \cup B_{d}\right| \leq\left|A_{1} \cup \cdots \cup A_{d}\right|=s+1 .
$$

In particular, $\left|S_{j i}\left(B_{1}\right) \cap \cdots \cap S_{j i}\left(B_{d}\right)\right| \leq t-1$ and $\left|S_{j i}\left(B_{1}\right) \cup \cdots \cup S_{j i}\left(B_{d}\right)\right|=s$, so $S_{j i}(\mathcal{B})$ is not $(d, s, t)$-conditionally intersecting, and it follows that $\mathcal{B}$ is $(j, i)$-unstable.

Note that $\mathcal{B} \neq S_{j i}(\mathcal{B})$. The only sets of $\mathcal{B}$ which change under $S_{j i}$ are those in $\mathcal{G}^{\prime}$. Thus, $\mathcal{G}^{\prime} \neq \emptyset$, so $\mathcal{G} \neq \mathcal{A}_{i \bar{j}}$.

By Lemma 11], $\mathcal{B}=\mathcal{B}_{\overline{j i}} \cup \mathcal{B}_{\overline{j i}} \cup \mathcal{B}_{\overline{j i}}$, where

$$
\begin{aligned}
\mathcal{B}_{\overline{j i}} & =\left\{B \in \mathcal{B}: i \in B, j \notin B, B^{\prime}=(B-\{i\}) \cup\{j\} \notin \mathcal{F}\right\} \\
& =\left\{B \in \mathcal{B}: S_{j i}(B) \neq B\right\} .
\end{aligned}
$$

Comparing this expression for $\mathcal{B}$ with the definition $\mathcal{B}=\mathcal{A}_{\bar{i} j} \cup \mathcal{A}_{\bar{i} \bar{j}} \cup\left(\mathcal{A}_{i \bar{j}}-\mathcal{G}\right) \cup \mathcal{G}^{\prime}$ yields the identities

$$
\mathcal{B}_{\bar{j} i}=\mathcal{A}_{i \bar{j}}-\mathcal{G}, \quad \mathcal{B}_{j \bar{i}}=\mathcal{A}_{\bar{i} j} \cup \mathcal{G}^{\prime} \quad \text { and } \quad \mathcal{B}_{\overline{j i}}=\mathcal{A}_{\bar{i} \bar{j}} .
$$

Define

$$
\mathcal{C}=\mathcal{B}_{\overline{j i}} \cup \mathcal{B}_{\overline{j i}} \cup\left(\mathcal{B}_{j \bar{i}}-\mathcal{H}\right) \cup \mathcal{H}^{\prime}
$$

We wish to show that $\mathcal{A}=\mathcal{C}$ and do this by showing that $\mathcal{H}=\mathcal{G}^{\prime}$ and $\mathcal{H}^{\prime}=\mathcal{G}$.

The elements $B$ of $\mathcal{G}^{\prime}$ are the sets of the form $B=(A-\{i\}) \cup\{j\}$ for some set $A \in \mathcal{G} \subseteq \mathcal{A}_{i \bar{j}}$. Since $(B-\{j\}) \cup\{i\}=A \in \mathcal{F}$, it follows that $S_{i j}(B)=B$. Thus, $\mathcal{H}=\mathcal{G}^{\prime}$ and hence

$$
\mathcal{H}^{\prime}=\left\{(B-\{j\}) \cup\{i\}: B \in \mathcal{G}^{\prime}\right\}=\mathcal{G} .
$$

Now by Lemma 11, every $A \in \mathcal{A}_{\bar{i} j}$ satisfies $S_{i j}(A) \neq A$, so $\mathcal{H} \cap \mathcal{A}_{\bar{i} j}=\emptyset$ and thus $\mathcal{G}^{\prime} \cap \mathcal{A}_{\overline{i j}}=\emptyset$. Hence by Lemma 11.

$$
\begin{aligned}
\mathcal{C} & =\mathcal{B}_{\overline{j i}} \cup \mathcal{B}_{\overline{j i}} \cup\left(\mathcal{B}_{\overline{j i}}-\mathcal{H}\right) \cup \mathcal{H}^{\prime} \\
& =\left(\left(\mathcal{A}_{i \bar{j}}-\mathcal{G}\right) \cup \mathcal{H}^{\prime}\right) \cup \mathcal{A}_{\overline{j i}} \cup\left(\left(\mathcal{A}_{\overline{i j}} \cup \mathcal{G}^{\prime}\right)-\mathcal{H}\right) \\
& =\left(\left(\mathcal{A}_{i \bar{j}}-\mathcal{G}\right) \cup \mathcal{G}\right) \cup \mathcal{A}_{\overline{j i}} \cup\left(\left(\mathcal{A}_{\bar{i} j} \cup \mathcal{G}^{\prime}\right)-\mathcal{G}^{\prime}\right) \\
& =\mathcal{A}_{i \bar{j}} \cup \mathcal{A}_{\overline{j i}} \cup \mathcal{A}_{\overline{i j}} \\
& =\mathcal{A} .
\end{aligned}
$$

By Proposition 3, the size of a $(d, 2 k)$-conditionally intersecting family $\mathcal{F} \subseteq\left(\begin{array}{c}{[n]} \\ k\end{array}\right)$ is preserved under shifting. Iterative shifts to $\mathcal{F}$ will result in a family $\mathcal{F}^{\prime}$ that is either stable or is $(i, j)$-unstable for some $i, j$; that is, a shift applied to $\mathcal{F}^{\prime}$ will result in a family that is not $(d, 2 k)$-conditionally intersecting.

An upper bound on the size of the former is determined by Theorem 5 . Thus to determine the maximum possible size of $(d, 2 k)$-conditionally intersecting families, it suffices to consider families that are $(i, j)$-unstable for some $i, j$. 


\section{Non-intersecting $(d, 2 k)$-conditionally intersecting families}

Theorem 1 by Frankl [6] provides an upper bound on the size of $d$-wise intersecting families. Muyabi's Conjecture, if true, would improve this result by providing an upper bound on size of families that are $(d, 2 k)$-conditionally intersecting. The two bounds - and the bound in Theorem [5- turn out to be identical, and this bound is indeed in each case achieved by star families.

To further sharpen Mubayi's Conjecture, it may be useful to distinguish more explicitly between the two conditions given by Frankl's theorem and Mubayi's Conjecture. In particular, it is worth considering the upper bounds on the size of families addressed by the former (i.e., $d$-wise intersecting families) and the families addressed by the latter (i.e., $(d, 2 k)$-conditionally intersecting families). Hence in this section, we consider families $\mathcal{F} \in\left(\begin{array}{c}{[n]} \\ k\end{array}\right)$ that are $(d, 2 k)$ conditionally intersecting but which are not $d$-wise intersecting; that is, for all distinct sets $A_{1}, \ldots, A_{d} \in \mathcal{F}$

$$
\begin{aligned}
& A_{1} \cap \cdots \cap A_{d} \neq \emptyset \text { whenever }\left|A_{1} \cup \cdots \cup A_{d}\right| \leq 2 k \\
& \text { but } A_{1} \cap \cdots \cap A_{d}=\emptyset \text { for at least some } d \text { distinct sets } A_{1}, \ldots, A_{d} \in \mathcal{F} .
\end{aligned}
$$

In particular, we pose the following conjecture.

Conjecture 13. For $k \geq d \geq 3$ and sufficiently large $n$, each family $\mathcal{F}$ that is $(d, 2 k)$ conditionally intersecting but which is not intersecting has size at most

$$
\left(\begin{array}{c}
n-k-1 \\
k-1
\end{array}\right)+1
$$

Furthermore, equality holds if and only if

$$
\mathcal{F}=\left\{A \in\left(\begin{array}{c}
{[n]} \\
k
\end{array}\right): x \in A \text { and } A \cap B=\emptyset\right\} \cup\{B\}
$$

for some fixed $x \in[n]$ and $B \in\left(\begin{array}{c}{[n]} \\ k\end{array}\right)$ such that $x \notin B$.

Note that the definition of the family $\mathcal{F}$ in the conjecture above differs from the maximallysized intersecting non-star family of the Hilton-Milner Theorem [12] (see also [8]), in which the condition $A \cap B=\emptyset$ is replaced by the negated condition $A \cap B \neq \emptyset$.

An indication that Conjecture 13 might be true is that the family $\mathcal{F}$ has size $\left(\begin{array}{c}n-k-1 \\ k-1\end{array}\right)+1$ which, asymptotically, converges to $\left(\begin{array}{l}n-1 \\ k-1\end{array}\right)$, the size of star families on $n$ elements which, in turn, are asymptotically the largest $(d, 2 k)$-conditionally intersecting families; see [11, 18].

However, Conjecture 13 would be false if $n$ were allowed to be small, since $\mathcal{F}$ would then be smaller than other non-intersecting $(d, 2 k)$-conditionally intersecting families. For instance, consider

$$
\mathcal{G}=\left\{B_{1}, B_{2}\right\} \cup\left\{A \in\left(\begin{array}{c}
{[n]} \\
k
\end{array}\right): x, y \in A, \text { and } A \cap\left([n]-\left(B_{1} \cup B_{2}\right)\right) \neq \emptyset\right\}
$$


where $B_{1}, B_{2} \in\left(\begin{array}{c}{[n]} \\ k\end{array}\right)$ are fixed disjoint sets, and $x \in B_{1}$ and $y \in B_{2}$ are fixed elements. The family $\mathcal{G}$ has size $\left(\begin{array}{c}n-2 \\ k-2\end{array}\right)-\left(\begin{array}{c}2 k-2 \\ k-2\end{array}\right)+2$ and is $(d, 2 k)$-intersecting, since the only disjoint sets in $\mathcal{G}$ are $B_{1}$ and $B_{2}$ and by definition any other member of $\mathcal{G}$ must contain an element not in $B_{1}$ or $B_{2}$. For $n=2 k+1, \mathcal{F}$ has size $k+1$ whereas $\mathcal{G}$ has size

$$
\left(\begin{array}{c}
(2 k+1)-2 \\
k-2
\end{array}\right)-\left(\begin{array}{c}
2 k-2 \\
k-2
\end{array}\right)+2=\left(\begin{array}{c}
2 k-1 \\
k-2
\end{array}\right)-\left(\begin{array}{c}
2 k-2 \\
k-2
\end{array}\right)+2=\left(\begin{array}{c}
2 k-2 \\
k-3
\end{array}\right)+2 .
$$

which is larger than $k+1$ for $k \geq 4$.

For completeness, let us consider families of pairs; that is, the case in which $k=2$. For this purpose, define a twin 2-star on $[n]$ to be any family $\mathcal{F} \subseteq\left(\begin{array}{c}{[n]} \\ 2\end{array}\right)$ consisting, for distinct and fixed $x, y \in[n]$, of $n-2$ unordered pairs $A \subseteq[n]$ of the form $\left\{z, z^{\prime}\right\}$ where $z \in[n]-\{x, y\}$ and $z^{\prime} \in\{x, y\}$ so that each element $z \in[n]-\{x, y\}$ appears exactly once in some set $A \in \mathcal{F}$. Note that twin stars generalise $\mathcal{F}$ in the conjecture for $k=2$.

Proposition 14. If $\mathcal{F} \subseteq\left(\begin{array}{c}{[n]} \\ 2\end{array}\right)$ is $(3,4)$-conditionally intersecting but is not intersecting, then

$$
|\mathcal{F}| \leq n-2,
$$

and equality holds if and only if $\mathcal{F}$ is a twin 2-star.

Proof. Suppose that $\mathcal{F}$ is $(3,4)$-conditionally intersecting but not intersecting; then for all distinct sets $A_{1}, A_{2}, A_{3} \in \mathcal{F}$

$$
A_{1} \cap A_{2} \cap A_{3} \neq \emptyset \text { whenever }\left|A_{1} \cup A_{2} \cup A_{3}\right| \leq 4 .
$$

Consider $\mathcal{F}$ to be the graph on $n$ vertices whose edges are the members of $\mathcal{F}$. Then (10) asserts that $\mathcal{F}$ contains no triangles or paths of length 3. Therefore, every connected component of $\mathcal{F}$ is a star. If there are $m$ connected components in $\mathcal{F}$, then there are $n-m$ edges in $\mathcal{F}$. Since $\mathcal{F}$ is not intersecting, $\mathcal{F}$ cannot be a star, so $m \geq 2$. Hence, $|\mathcal{F}| \leq n-2$ and equality holds only if $\mathcal{F}$ is a twin 2 -star.

\section{On $(d, s)$-conditionally intersecting families for $s<2 k$}

In this section, we review and extend some of the work by Frankl and Füredi [10] on $(d, s)$ conditionally intersecting families for $s<2 k$. Partition $[n]$ into $k$ almost equal parts $X_{1}, \ldots, X_{k}$ so that $\left\lfloor\frac{n}{k}\right\rfloor \leq\left|X_{i}\right| \leq\left\lfloor\frac{n}{k}\right\rfloor+1$, and define

$$
\mathcal{H}_{k}=\left\{A \in\left(\begin{array}{c}
{[n]} \\
k
\end{array}\right):\left|A \cap X_{i}\right|=1 \text { for all } i=1, \ldots, k\right\} .
$$

Frankl and Füredi [10] proved that this family is $(3,2 k-1)$-conditionally intersecting and of order $\Theta\left(n^{k}\right)$. Thus for fixed $k, \mathcal{H}_{k}$ is larger than a star for sufficiently large $n$. This shows that Mubayi's Conjecture cannot be extended to hold for $(d, s)$-conditionally intersecting families for $s<2 k$. Motivated by these facts, Frankl and Füredi [10] conjectured that $\mathcal{H}_{k}$ is, up to isomorphism, uniquely largest among $(3,2 k-1)$-conditionally intersecting families on $[n]$, at least for sufficiently large $n$. We extend this conjecture as follows. 
Conjecture 15. If $\mathcal{F} \subseteq\left(\begin{array}{c}{[n]} \\ k\end{array}\right)$ is $(d, 2 k-1)$-conditionally intersecting, then, for sufficiently large $n$,

$$
|\mathcal{F}| \leq\left|\mathcal{H}_{k}\right|
$$

Furthermore, equality holds if and only if $\mathcal{F}=\mathcal{H}_{k}$ up to isomorphism.

The family $\mathcal{H}_{k}$ can be generalised in the following way. Partition $[n]$ into $r \leq k$ fixed parts (not necessarily near-equal) $X_{1}, \ldots, X_{r}$. Choose fixed non-negative integers $x_{1}, \ldots, x_{r}$ such that $\sum_{i=1}^{r} x_{i} \leq k$ and define

$$
\mathcal{G}_{r}=\left\{A \in\left(\begin{array}{c}
{[n]} \\
k
\end{array}\right):\left|A \cap X_{i}\right| \geq x_{i} \text { for all } i=1, \ldots, r\right\} .
$$

Several maximal families from Extremal Set Theory can be expressed in this form.

Example 16. Stars are the unique maximum families for the Erdős-Ko-Rado Theorem for $n>2 k$ and for Theorem 1. Each star can be expressed as follows for some $y \in[n]$ :

$$
\left\{A \in\left(\begin{array}{c}
{[n]} \\
k
\end{array}\right): y \in F\right\}=\left\{A \in\left(\begin{array}{c}
{[n]} \\
k
\end{array}\right):\left|A \cap X_{1}\right| \geq x_{1} \text { and }\left|A \cap X_{2}\right| \geq x_{2}\right\}
$$

where $X_{1}=\{y\}$ and $X_{2}=[n]-\{y\}$ partition $[n]$ and where $x_{1}=1$ and $x_{2}=0$.

Example 17. Define $X_{1}=[t+2 j]$ and $X_{2}=[n]-X_{1}$ and $x_{1}=t+j$ and $x_{2}=0$ for some integers $t \geq 1$ and $j \geq 0$. The families

$$
\mathcal{F}_{j}=\left\{A \in\left(\begin{array}{c}
{[n]} \\
k
\end{array}\right):|A \cap[t+2 j]| \geq t+j\right\}=\left\{A \in\left(\begin{array}{c}
{[n]} \\
k
\end{array}\right):\left|A \cap X_{i}\right| \geq x_{i} \text { for } i=1,2\right\}
$$

have be shown in [2] to be the largest $t$-intersecting families $\mathcal{F} \subseteq\left(\begin{array}{c}{[n]} \\ k\end{array}\right)$.

Example 18. Define $X_{1}=[t], X_{2}=[k+1]-[t], X_{3}=[n]-X_{1}-X_{2}, x_{1}=t, x_{2}=1$ and $x_{3}=0$ for some integers $k, t \geq 1$. The family $\mathcal{F}_{1}$ from Example 17 and the family

$$
\begin{aligned}
\mathcal{F}^{\prime} & =\left\{A \in\left(\begin{array}{c}
{[n]} \\
k
\end{array}\right):[t] \subseteq A,([k+1]-[t]) \cap A \neq \emptyset\right\} \cup\{[k+1]-\{i\}: i \in[k+1]\} \\
& =\left\{A \in\left(\begin{array}{c}
{[n]} \\
k
\end{array}\right):\left|A \cap X_{i}\right| \geq x_{i} \text { for all } i=1,2,3\right\} \cup\{[k+1]-\{i\}: i \in[k+1]\}
\end{aligned}
$$

have been shown in [1] to be, up to isomorphism, the maximum $t$-intersecting families that satisfy $\left|\bigcap_{A \in \mathcal{F}} A\right|<t$.

Lemma 19. If $n>k \geq 2, d \geq 2$ and $\mathcal{F}=\left\{A \in 2^{[n]}:|A| \geq k\right\}$ contains $d$ sets $A_{1}, \ldots, A_{d}$ such that $A_{1} \cap \cdots \cap A_{d}=\emptyset$, then

$$
n \geq\left\lceil\frac{d k}{d-1}\right\rceil
$$


Proof. If $A_{1}, \ldots, A_{d} \in \mathcal{F}$ and $A_{1} \cap \cdots \cap A_{d}=\emptyset$, then by removing elements from each set $A_{i}$ of size greater than $k$, we obtain $d$ sets, $A_{1}^{\prime}, \ldots, A_{d}^{\prime} \in\left(\begin{array}{c}{[n]} \\ k\end{array}\right)$, such that $A_{1}^{\prime} \cap \cdots \cap A_{d}^{\prime}=\emptyset$. Hence,

$$
n(d-1) \geq \sum_{a \in[n]}\left|\left\{i \in[d]: a \in A_{i}^{\prime}\right\}\right|=\sum_{i \in[d]}\left|A_{i}^{\prime}\right| \geq d k,
$$

so $n \geq\left\lceil\frac{d k}{d-1}\right\rceil$.

We now determine the values of $d$ and $s$ for which $\mathcal{G}_{r}$ is a $(d, s)$-conditionally intersecting family. If $\mathcal{G}_{r}$ is $d$-wise intersecting, then it is trivially $(d, s)$-conditionally intersecting for any $s$. The following theorem completes the determination of the values of $d$ and $s$.

Theorem 20. If the family $\mathcal{G}_{r}$ is not $d$-wise intersecting, then it is $(d, s)$-conditionally intersecting but not $(d, s+1)$-conditionally intersecting, where

$$
s=\max \left\{\left\lceil\frac{d k}{d-1}\right\rceil-1, \sum_{i=1}^{r}\left\lceil\frac{d x_{i}}{d-1}\right\rceil-1\right\} .
$$

Proof. Suppose that the family $\mathcal{G}_{r}$ is not $d$-wise intersecting, and let $s$ be defined as above. Let $A_{1}, \ldots, A_{d} \in \mathcal{G}_{r}$ be $d$ sets with empty intersection. For each $i, A_{j} \cap X_{i}$ is a subset of $\left(A_{1} \cup \cdots \cup A_{d}\right) \cap X_{i}$ of size at least $x_{i}$. Also,

$$
\left(A_{1} \cap X_{i}\right) \cap \cdots \cap\left(A_{d} \cap X_{i}\right) \subseteq A_{1} \cap \cdots \cap A_{d}=\emptyset .
$$

Hence by Lemma 19

$$
\left|\left(A_{1} \cup \cdots \cup A_{d}\right) \cap X_{i}\right| \geq\left\lceil\frac{d x_{i}}{d-1}\right\rceil .
$$

The sets $X_{1}, \ldots, X_{r}$ partition $[n]$, so

$$
\left|A_{1} \cup \cdots \cup A_{d}\right|=\sum_{i=1}^{r}\left|\left(A_{1} \cup \cdots \cup A_{d}\right) \cap X_{i}\right| \geq \sum_{i=1}^{r}\left\lceil\frac{d x_{i}}{d-1}\right\rceil .
$$

Therefore, $\mathcal{G}_{r}$ is $\left(d, \sum_{i=1}^{r}\left\lceil\frac{d x_{i}}{d-1}\right\rceil-1\right)$-conditionally intersecting. Also by Lemma 19,

$$
\left|A_{1} \cup \cdots \cup A_{d}\right| \geq\left\lceil\frac{d k}{d-1}\right\rceil .
$$

Thus, $\mathcal{G}_{r}$ is $\left(d,\left\lceil\frac{d k}{d-1}\right\rceil-1\right)$-conditionally intersecting and thus $(d, s)$-conditionally intersecting.

We now show that $\mathcal{G}_{r}$ is not $(d, s+1)$-conditionally intersecting by finding $d$ sets in $\mathcal{G}_{r}$ with empty intersection and union of size $s+1$. For each $i$, define

$$
y_{i}:=\left\lceil\frac{d x_{i}}{d-1}\right\rceil \text {. }
$$

As $\mathcal{G}_{r}$ is not $d$-wise intersecting, there are $d$ members $A_{1}, \ldots, A_{d}$ of $\mathcal{G}_{r}$ with empty intersection. Then by (11), $\left|X_{i}\right| \geq y_{i}$ for all $i$. Choose $X_{i}^{\prime} \subseteq X_{i}$ such that $\left|X_{i}^{\prime}\right|=y_{i}$ and let $a_{i}$ be any integer 
satisfying $d x_{i} \leq a_{i} \leq(d-1) y_{i}$. We will now construct $d$ subsets $A_{i, 1}, \ldots, A_{i, d} \subseteq X_{i}^{\prime}$ each of size $x_{i}$ or $x_{i}+1$ and satisfying $A_{i, 1} \cap \cdots \cap A_{i, d}=\emptyset$ and

$$
\sum_{j=1}^{d}\left|A_{i, j}\right|=a_{i}
$$

First, set $A_{i, 1}=\cdots=A_{i, d}=\emptyset$. Add an arbitrary element $x \in X_{i}^{\prime}$ to $d-1$ of the sets $A_{i, j}$. Choose a new element $x^{\prime} \in X_{i}^{\prime}$ and add it to $d-1$ of the smallest of the sets $A_{i, j}$. Continue to add new elements to $d-1$ of the smallest sets until all but one element of $X_{i}^{\prime}$ have been added. Add the remaining element to $a_{i}-(d-1)\left(y_{i}-1\right)$ of the smallest of the sets $A_{i, j}$. The resulting sets $A_{i, 1}, \ldots, A_{i, d}$ satisfy $A_{i, 1} \cap \cdots \cap A_{i, d}=\emptyset$ as no element was added to all of the sets $A_{i, j}$. Also,

$$
\sum_{j=1}^{d}\left|A_{i, j}\right|=\sum_{x \in X_{i}^{\prime}}\left|\left\{j: x \in A_{i, j}\right\}\right|=(d-1)\left(y_{i}-1\right)+a_{i}-(d-1)\left(y_{i}-1\right)=a_{i} .
$$

Finally, the sets $A_{i, 1}, \ldots, A_{i, d}$ are of near-equal size and as

$$
d x_{i} \leq \sum_{j=1}^{d}\left|A_{i, j}\right| \leq(d-1) y_{i}<d\left(x_{i}+1\right),
$$

each set $A_{i, j}$ has size $x_{i}$ or $x_{i+1}$.

Given an integer $a$ with $\sum_{i=1}^{r} d x_{i} \leq a \leq \sum_{i=1}^{r}(d-1) y_{i}$, we construct new sets $A_{1}, \ldots, A_{d}$ of near-equal size that satisfy $A_{1} \cap \cdots \cap A_{d}=\emptyset, x_{i} \leq\left|A_{j} \cap X_{i}\right| \leq x_{i}+1$ for all $i$ and $j$, and

$$
\sum_{j=1}^{d}\left|A_{j}\right|=a
$$

Let $a_{1}, \ldots, a_{r}$ be integers such that $d x_{i} \leq a_{i} \leq(d-1) y_{i}$ and $a_{1}+\cdots+a_{r}=a$. Construct $A_{i, 1}, \ldots, A_{i, d}$ as above for all $i$, and set $A_{j}=A_{1, j}$ for all $j$. Relabel the $j$ indices so that the indices $j$ for which $\left|A_{2, j}\right|=x_{2}+1$ are also the indices $j$ for which $A_{j}$ are the smallest of the sets $A_{1}, \ldots, A_{d}$. Now add the elements of $A_{2, j}$ to $A_{j}$ for all $j$; i.e., let $A_{j}=A_{1, j} \cup A_{2, j}$. Again, relabel the sets so that $\left|A_{3, j}\right|=x_{3}+1$ for the indices $j$ for which the sets $A_{j}$ are the smallest of the sets $A_{1}, \ldots, A_{d}$. Continue to add $A_{i, j}$ and relabel in this way, for $i=3, \ldots, r$ and all $j$. We have thus constructed sets

$$
A_{j}=\bigcup_{i=1}^{r} A_{i, j}
$$

for all $j$. Since $A_{i, 1} \cap \cdots \cap A_{i, d}=\emptyset$ for all $i$, it follows that $A_{1} \cap \cdots \cap A_{d}=\emptyset$. Note that $x_{i} \leq\left|A_{j} \cap X_{i}\right|=\left|A_{i, j}\right| \leq x_{i}+1$ for all $i$ and $j$, and that, by construction, the sets $A_{j}$ are near-equally sized and that

$$
\sum_{j=1}^{d}\left|A_{j}\right|=\sum_{j=1}^{d} \sum_{i=1}^{r}\left|A_{i, j}\right|=\sum_{i=1}^{r} \sum_{j=1}^{d}\left|A_{i, j}\right|=\sum_{i=1}^{r} a_{i}=a .
$$


We now wish to prove that, for some $a$, the sets $A_{1}, \ldots, A_{d}$ constructed in the way described above are in $\mathcal{G}_{r}$ and that the union of the sets $A_{1}, \ldots, A_{d}$ has size $s+1$. For this purpose, we consider two cases, namely the case in which $\sum_{i=1}^{r}(d-1) y_{i}$ is greater than $d k$, and the cases in which it is not.

Case I: Suppose that $\sum_{i=1}^{r}(d-1) y_{i} \geq d k$. Then

$$
\sum_{i=1}^{r} y_{i}=\max \left\{\left\lceil\frac{d k}{d-1}\right\rceil, \sum_{i=1}^{r} y_{i}\right\}=\max \left\{\left\lceil\frac{d k}{d-1}\right\rceil, \sum_{i=1}^{r}\left\lceil\frac{d x_{i}}{d-1}\right\rceil\right\}=s+1 .
$$

Also,

$$
\sum_{i=1}^{r} d x_{i} \leq d k \leq \sum_{i=1}^{r}(d-1) y_{i}
$$

so we were allowed to choose the value $a=d k$ in the above construction, and thus obtain the sets $A_{1}, \ldots, A_{d}$ of near-equal size satisfying

$$
\sum_{i=1}^{d}\left|A_{i}\right|=a=d k
$$

Since $\left|A_{j}\right| \leq k$ for each $j$, it follows that $\left|A_{1}\right|=\cdots=\left|A_{d}\right|=k$. Since $\left|A_{j} \cap X_{i}\right| \geq x_{i}$ for all $i$ and $j$, it follows that $A_{1}, \ldots, A_{d} \in \mathcal{G}_{r}$. By construction,

$$
\left|A_{1} \cup \ldots \cup A_{d}\right|=\sum_{i=1}^{r}\left|X_{i}^{\prime}\right|=\sum_{i=1}^{r} y_{i}=s+1 .
$$

CASE II: Now suppose that $\sum_{i=1}^{r}(d-1) y_{i}<d k$. Then

$$
s=\max \left\{\left\lceil\frac{d k}{d-1}\right\rceil-1, \sum_{i=1}^{r}\left\lceil\frac{d x_{i}}{d-1}\right\rceil-1\right\}=\max \left\{\left\lceil\frac{d k}{d-1}\right\rceil-1, \sum_{i=1}^{r} y_{i}-1\right\}=\left\lceil\frac{d k}{d-1}\right\rceil-1 .
$$

Also, we may assume that the value $a=\sum_{i=1}^{r}(d-1) y_{i}$ was used in the above construction, so the resulting sets $A_{1}, \ldots, A_{d}$ satisfy

$$
\sum_{i=1}^{d}\left|A_{i}\right|=a=\sum_{i=1}^{r}(d-1) y_{i} .
$$

Since $a_{i} \leq(d-1) y_{i}$ for each $i$ and $a_{1}+\cdots a_{r}=a=\sum_{i=1}^{r}(d-1) y_{i}$, it follows that $a_{i}=(d-1) y_{i}$ for each $i$. Hence in the construction, each element in $X^{\prime}:=X_{1}^{\prime} \cup \cdots \cup X_{r}^{\prime}$ was added to exactly $d-1$ of the sets $A_{1}, \ldots, A_{d}$ or, in the case of the $r$ final elements, to

$$
a_{i}-(d-1)\left(y_{i}-1\right)=(d-1) y_{i}-(d-1)\left(y_{i}-1\right)=d-1
$$

of the sets. That is, each element of $X^{\prime}$ lies in exactly $d-1$ of the sets $A_{1}, \ldots, A_{d}$. Add an element of $[n]-X^{\prime}$ to $d-1$ of the smallest sets $A_{i}$ and continue to add elements of $[n]-X^{\prime}$ in this way until

$$
d k \leq \sum_{i=1}^{d}\left|A_{i}\right|<d k+d-1 .
$$


This is possible, for suppose by way of contradiction that it were not; then every element of $[n]$ would lie in exactly $d-1$ of the sets $A_{1}, \ldots, A_{d}$ and yet $\sum_{i=1}^{d}\left|A_{i}\right|<d k$. Then

$$
(d-1) n=\sum_{j \in[n]}\left|\left\{A_{i}: j \in A_{i}\right\}\right|=\sum_{i=1}^{d}\left|A_{i}\right|<d k .
$$

However, $\mathcal{G}_{r}$ is not $d$-wise intersecting, so $n \geq\left\lceil\frac{d k}{d-1}\right\rceil$ by Lemma 19, a contradiction.

Now remove an element from a largest set $A_{i}$ and continue to do so until

$$
\sum_{i=1}^{d}\left|A_{i}\right|=d k
$$

As in CASE I, the sets $A_{1}, \ldots, A_{d}$ have near-equal size and $\left|A_{j}\right| \leq k$ for each $j$, so $\left|A_{1}\right|=\cdots=$ $\left|A_{d}\right|=k$. As $\left|A_{j} \cap X_{i}\right| \geq x_{j}$ for all $i$ and $j$, it follows that $A_{1}, \ldots, A_{d} \in \mathcal{G}_{r}$. The number of elements of $[n]-X^{\prime}$ added to the sets $A_{1}, \ldots, A_{d}$ is

$$
\left\lceil\frac{d k-\sum_{i=1}^{r}(d-1) y_{i}}{d-1}\right\rceil=\left\lceil\frac{d k}{d-1}\right\rceil-\sum_{i=1}^{r} y_{i}
$$

and the number of elements of $X^{\prime}=X_{1}^{\prime} \cup \cdots \cup X_{r}^{\prime}$ added to the sets $A_{1}, \ldots, A_{d}$ is $\sum_{i=1}^{r} y_{i}$, so

$$
\left|A_{1} \cup \cdots \cup A_{d}\right|=\left\lceil\frac{d k}{d-1}\right\rceil-\sum_{i=1}^{r}\left\lceil\frac{d k}{d-1}\right\rceil+\sum_{i=1}^{r} y_{i}=\left\lceil\frac{d k}{d-1}\right\rceil=s+1 .
$$

In both CASE I and CASE II, we have $d$ sets $A_{1}, \ldots, A_{d} \in \mathcal{G}_{r}$ with union size $s+1$ and yet empty mutual intersection. Hence, the sets $A_{1}, \ldots, A_{d}$ lie in $\mathcal{G}_{r}$ and are not $(d, s+1)$-conditionally intersecting, so neither is $\mathcal{G}_{r}$.

Example 21. By Theorem $\left[20\right.$, the family $\mathcal{F}_{j}$ from Example 17 is $\left(d,\left\lceil\frac{d k}{d-1}\right\rceil-1\right)$-conditionally intersecting for all $d \geq 3$ but is not $\left(d,\left\lceil\frac{d k}{d-1}\right\rceil\right)$-conditionally intersecting.

\section{On $(2, s)$-conditionally intersecting families of $2^{[n]}$ : Part I}

Erdős et al. [5] proved the following bound on the size of each intersecting family $\mathcal{F} \subseteq 2^{[n]}$.

Lemma 22. [5] If $\mathcal{F} \subseteq 2^{[n]}$ is intersecting, then

$$
|\mathcal{F}| \leq 2^{n-1} .
$$

A star meets the bound above but is not the unique maximum family. While weak asymptotic results are known (see [3]), it is a difficult challenge to classify the case of equality for general $n$. It is also difficult to count the number of families attaining the bound of Lemma 22 , even for small $n$. The highest value of $n$ for which such counting has presently been achieved is 9 ; see [3]. 
The aim of this section and that of Section 7 is to extend Lemma 22, To this aim, we extend the $(2, s)$-conditionally intersecting condition on families $\mathcal{F} \subseteq\left(\begin{array}{c}{[n]} \\ k\end{array}\right)$ to families $\mathcal{F} \subseteq 2^{[n]}$. In particular, a family $\mathcal{F} \subseteq 2^{[n]}$ is $(2, s)$-conditionally intersecting if

$$
A \cap B \neq \emptyset \quad \text { whenever } \quad|A \cup B| \leq s \quad \text { for each } A, B \in \mathcal{F} .
$$

Theorem 23 below provides tight upper bounds on the sizes of $(2, s)$-conditionally intersecting families $\mathcal{F} \subseteq 2^{[n]}$, and describing precisely the families that attain these bounds. This theorem extends Lemma 22, the latter is obtained from the former by setting $s=n$.

Theorem 23. Suppose that $\mathcal{F} \subseteq 2^{[n]}$ is $(2, s)$-conditionally intersecting for some $s \leq n$. If $s=2 k$, then

$$
|\mathcal{F}| \leq\left(\begin{array}{l}
n-1 \\
k-1
\end{array}\right)+\sum_{i=k+1}^{n}\left(\begin{array}{l}
n \\
i
\end{array}\right)
$$

and if $s<n$ also holds, then equality holds above if and only if, up to isomorphism,

$$
\mathcal{F}=\left\{F \in\left(\begin{array}{c}
{[n]} \\
k
\end{array}\right): 1 \in F\right\} \cup\left(\begin{array}{c}
{[n]} \\
>k
\end{array}\right) \text {. }
$$

If $s=2 k-1$, then

$$
|\mathcal{F}| \leq \sum_{i=k}^{n}\left(\begin{array}{l}
n \\
i
\end{array}\right)
$$

and if $s<n$ also holds, then equality holds above if and only if $\mathcal{F}=\left(\begin{array}{c}{[n]} \\ \geq k\end{array}\right)$.

Here, $\left(\begin{array}{c}{[n]} \\ >k\end{array}\right)$ and $\left(\begin{array}{c}{[n]} \\ \geq k\end{array}\right)$ denote the respective families

$$
\left(\begin{array}{c}
{[n]} \\
>k
\end{array}\right)=\left\{F \in 2^{[n]}:|F|>k\right\} \quad \text { and } \quad\left(\begin{array}{c}
{[n]} \\
\geq k
\end{array}\right)=\left\{F \in 2^{[n]}:|F| \geq k\right\} .
$$

Note that Keevash and Mubayi [14] also presented a conditionally intersecting definition with the same terminology as ours; their definition is however different from that given here, and their results are only tangentially related to the results of this section.

In order to prove Theorem 23, we require some preliminary definitions and results. For a family $\mathcal{F} \subseteq\left(\begin{array}{c}{[n]} \\ k\end{array}\right)$, define

$$
\mathcal{F}^{C}=\left\{F^{C}: F \in \mathcal{F}\right\} \quad \text { and } \quad \mathcal{F}_{r}=\{F \in \mathcal{F}:|F|=r\} .
$$

Also, for each $\ell \leq k$, define the $\ell$-shadow of $\mathcal{F}$ to be the set

$$
\sigma_{\ell}(\mathcal{F})=\left\{F \in\left(\begin{array}{c}
{[n]} \\
\ell
\end{array}\right): F \subseteq A \text { for some } A \in \mathcal{F}\right\} .
$$

The following theorem by Katona [13] gives a lower bound for the size of a shadow under certain conditions. 
Theorem 24. [13] If $0 \leq k-t \leq \ell \leq k$ and $\mathcal{F} \subseteq\left(\begin{array}{c}{[n]} \\ k\end{array}\right)$ is $t$-intersecting, i.e., $|A \cap B| \geq t$ for all $A, B \in \mathcal{F}$, then

$$
\left|\sigma_{\ell}(\mathcal{F})\right| \geq \frac{\left(\begin{array}{c}
2 k-t \\
\ell
\end{array}\right)}{\left(\begin{array}{c}
2 k-t \\
k
\end{array}\right)}|\mathcal{F}| .
$$

Furthermore, equality holds if and only if $n=2 k-t, \mathcal{F}=\left(\begin{array}{c}{[n]} \\ k\end{array}\right)$ and $\ell \in\{k, k-t\}$.

We are now ready to prove Theorem 23 and will follow an approach similar to that in [13].

Proof of Theorem [23. First suppose that $\mathcal{F}$ contains no set of size less than $k=\left\lceil\frac{s}{2}\right\rceil$. Suppose that $s=2 k$ and define

$$
\mathcal{F}^{+}=\mathcal{F} \cup\left(\begin{array}{c}
{[n]} \\
>k
\end{array}\right)=\mathcal{F}_{k} \cup\left(\begin{array}{c}
{[n]} \\
>k
\end{array}\right) \text {. }
$$

If $A, B \in \mathcal{F}_{k}$ are distinct sets in $\mathcal{F}$, then $|A \cup B| \leq 2 k=s$, so $A \cap B \neq \emptyset$ since $\mathcal{F}$ is $(2, s)$-conditionally intersecting. Hence, $\mathcal{F}_{k}$ is an intersecting family, so by the Erdös-KoRado Theorem [5],

$$
|\mathcal{F}| \leq\left|\mathcal{F}^{+}\right|=\left|\mathcal{F}_{k}\right|+\left|\left(\begin{array}{c}
{[n]} \\
>k
\end{array}\right)\right| \leq\left(\begin{array}{c}
n-1 \\
k-1
\end{array}\right)+\sum_{i=k+1}^{n}\left(\begin{array}{l}
n \\
i
\end{array}\right),
$$

and the unique maximum family (up to isomorphism) is

$$
\mathcal{F}=\left\{F \in\left(\begin{array}{c}
{[n]} \\
k
\end{array}\right): 1 \in F\right\} \cup\left(\begin{array}{c}
{[n]} \\
>k
\end{array}\right) .
$$

If $s=2 k-1$, then $\mathcal{F} \subseteq\left(\begin{array}{c}{[n]} \\ \geq k\end{array}\right)$, so

$$
|\mathcal{F}| \leq \sum_{i=k}^{n}\left(\begin{array}{l}
n \\
i
\end{array}\right)
$$

and the unique maximum family is $\left(\begin{array}{c}{[n]} \\ \geq k\end{array}\right)$.

Now suppose that $\mathcal{F}_{r^{\prime}} \neq \emptyset$ for some positive integer $r^{\prime}<k$ and define

$$
\mathcal{F}^{\prime}=\left(\mathcal{F} \cup \bigcup_{r=1}^{k-1} \sigma_{s-r}\left(\left(\mathcal{F}_{r}\right)^{C}\right)\right)-\bigcup_{r=1}^{k-1} \mathcal{F}_{r}
$$

We first show that $|\mathcal{F}| \leq\left|\mathcal{F}^{\prime}\right|$. If $A \in \sigma_{s-r}\left(\left(\mathcal{F}_{r}\right)^{C}\right)$, then $A \subseteq F^{C}$ for some $F \in \mathcal{F}_{r}$, so

$$
F \cap A=\emptyset \quad \text { and } \quad|A \cup F|=|A|+|F|=(s-r)+r=s .
$$

Hence, $A \notin \mathcal{F}$ since $F \in \mathcal{F}$ and $\mathcal{F}$ is $(2, s)$-conditionally intersecting; therefore, $\mathcal{F} \cap \sigma_{s-r}\left(\left(\mathcal{F}_{r}\right)^{C}\right)=$ $\emptyset$. For any $A, B \in\left(\mathcal{F}_{r}\right)^{C}$,

$$
|A \cap B|=|A|+|B|-|A \cup B| \geq 2(n-r)-n=n-2 r,
$$

so $\left(\mathcal{F}_{r}\right)^{C}$ is $(n-2 r)$-intersecting. As $s-r \geq r=(n-r)-(n-2 r)$, Theorem 24 implies that

$$
\left|\sigma_{s-r}\left(\left(\mathcal{F}_{r}\right)^{C}\right)\right| \geq \frac{\left(\begin{array}{c}
n \\
s-r
\end{array}\right)}{\left(\begin{array}{c}
n \\
n-r
\end{array}\right)}\left|\left(\mathcal{F}_{r}\right)^{C}\right|=\frac{\left(\begin{array}{c}
n \\
s-r
\end{array}\right)}{\left(\begin{array}{c}
n \\
n-r
\end{array}\right)}\left|\mathcal{F}_{r}\right| .
$$


Now, $s-r \geq r$, so if $s-r \leq \frac{n}{2}$, then $\left(\begin{array}{c}n \\ s-r\end{array}\right) \geq\left(\begin{array}{c}n \\ r\end{array}\right)=\left(\begin{array}{c}n \\ n-r\end{array}\right)$. Otherwise, $\frac{n}{2} \leq s-r \leq n-r$; also in this case, $\left(\begin{array}{c}n \\ s-r\end{array}\right) \geq\left(\begin{array}{c}n \\ n-r\end{array}\right)$. Hence, $\left|\sigma_{s-r}\left(\left(\mathcal{F}_{r}\right)^{C}\right)\right| \geq\left|\mathcal{F}_{r}\right|$, so

$$
\left|\mathcal{F}^{\prime}\right|=|\mathcal{F}|+\sum_{r=1}^{k-1}\left|\sigma_{s-r}\left(\left(\mathcal{F}_{r}\right)^{C}\right)\right|-\sum_{r=1}^{k-1}\left|\mathcal{F}_{r}\right|=|\mathcal{F}|+\sum_{r=1}^{k-1}\left(\left|\sigma_{s-r}\left(\left(\mathcal{F}_{r}\right)^{C}\right)\right|-\left|\mathcal{F}_{r}\right|\right) \geq|\mathcal{F}|
$$

Next, we prove that $\mathcal{F}^{\prime}$ is $(2, s)$-conditionally intersecting. Suppose that $|A \cup B| \leq s$ for $A, B \in$ $\mathcal{F}^{\prime}$. If $A, B \in \mathcal{F}$, then $A \cap B \neq \emptyset$ since $\mathcal{F}$ is $(2, s)$-conditionally intersecting; otherwise, either $A$ or $B$ is an element of $\mathcal{F}^{\prime}-\mathcal{F}=\bigcup_{r=1}^{k-1} \sigma_{s-r}\left(\left(\mathcal{F}_{r}\right)^{C}\right)$, without loss of generality $A \in \sigma_{s-r}\left(\left(\mathcal{F}_{r}\right)^{C}\right)$. Then

$$
|A \cap B|=|A|+|B|-|A \cup B| \geq(s-r)+k-s=k-r>0,
$$

so $A \cap B \neq \emptyset$. We conclude that $\mathcal{F}^{\prime}$ is $(2, s)$-conditionally intersecting.

Since $\mathcal{F}^{\prime}$ contains no set of size smaller than $k$, the first part of the proof implies that

$$
|\mathcal{F}| \leq\left|\mathcal{F}^{\prime}\right| \leq \sum_{i=k}^{n}\left(\begin{array}{l}
n \\
i
\end{array}\right)
$$

when $s=2 k-1$ and that, when $s=2 k$,

$$
|\mathcal{F}| \leq\left|\mathcal{F}^{\prime}\right| \leq\left(\begin{array}{l}
n-1 \\
k-1
\end{array}\right)+\sum_{i=k+1}^{n}\left(\begin{array}{l}
n \\
i
\end{array}\right)
$$

Finally, assume that bounds above met with $s<n$ and that $\mathcal{F}_{r} \neq \emptyset$ for some $r<k$. By the proof above, it follows that

$$
\left|\sigma_{s-r}\left(\left(\mathcal{F}_{r}\right)^{C}\right)\right|=\frac{\left(\begin{array}{c}
n \\
s-r
\end{array}\right)}{\left(\begin{array}{c}
n \\
n-r
\end{array}\right)}\left|\left(\mathcal{F}_{r}\right)^{C}\right|
$$

so Theorem 24 implies that $\mathcal{F}_{r}^{C}=\left(\begin{array}{c}{[n]} \\ n-r\end{array}\right)$ and so $\mathcal{F}_{r}=\left(\begin{array}{c}{[n]} \\ r\end{array}\right)$. However, $n>2 r$, so $\mathcal{F}_{r}=\left(\begin{array}{c}{[n]} \\ r\end{array}\right)$ is not intersecting, so there are disjoint sets $A, B \in \mathcal{F}$ with $|A \cup B|=2 r \leq s$, contradicting that $\mathcal{F}$ is $(2, s)$-conditionally intersecting. Hence when $s<n$ and $\mathcal{F}$ contains sets of size less than $k,|\mathcal{F}|$ cannot reach its upper bound. This concludes the proof.

\section{On $(2, s)$-conditionally intersecting families of $2^{[n]}$ : Part II}

In the previous section, we extended Lemma 22 by presenting and proving the more general Theorem 23. In this final section, we will extend this line of investigation further, by expanding the focus on $2^{[n]}$ to the more general set of families

$$
\left(\begin{array}{c}
{[n]} \\
\leq u
\end{array}\right)=\{X \subseteq[n]:|X| \leq u\}
$$

for certain values of $u$, as follows. 
Theorem 25. Let $\mathcal{F} \subseteq\left(\begin{array}{c}{[n]} \\ \leq u\end{array}\right)$ be $(2, s)$-conditionally intersecting for some $s \leq n$.

(i) If $u \geq s-1$ and $s=2 k$, then

$$
|\mathcal{F}| \leq\left(\begin{array}{l}
n-1 \\
k-1
\end{array}\right)+\sum_{i=k+1}^{u}\left(\begin{array}{l}
n \\
i
\end{array}\right)
$$

and if $s<n$ also holds, then equality holds if and only if, up to isomorphism,

$$
\mathcal{F}=\left\{F \in 2^{[n]}: k<|F| \leq u\right\} \cup\left\{F \in\left(\begin{array}{c}
{[n]} \\
k
\end{array}\right): 1 \in F\right\} .
$$

(ii) If $u \geq s-1$ and $s=2 k-1$, then

$$
|\mathcal{F}| \leq \sum_{i=k}^{u}\left(\begin{array}{l}
n \\
i
\end{array}\right)
$$

and if $s<n$ also holds, then equality holds above if and only if

$$
\mathcal{F}=\left\{F \in 2^{[n]}: k \leq|F| \leq u\right\} .
$$

(iii) If $u \leq k=\left\lfloor\frac{s}{2}\right\rfloor$, then

$$
|\mathcal{F}| \leq \sum_{r=1}^{u}\left(\begin{array}{l}
n-1 \\
r-1
\end{array}\right)
$$

and equality holds if and only if, up to isomorphism,

$$
\mathcal{F}=\left\{F \in 2^{[n]}: k \leq|F| \leq u \text { and } 1 \in F\right\} .
$$

Proof. The first two parts of the theorem follow easily from the proof of Theorem 23 and the observation that one may freely add or remove sets of size at least $s$ without violating the $(2, s)$-conditionally intersecting condition. Suppose therefore that $u \leq k=\left\lfloor\frac{s}{2}\right\rfloor$. Then $\mathcal{F}$ is intersecting; in particular, the subfamilies

$$
\mathcal{F}_{r}=\{F \in \mathcal{F}:|F|=r\}
$$

are intersecting (so $\left|\mathcal{F}_{1}\right| \leq 1$ ). By the Erdös-Ko-Rado Theorem [5], each family $\mathcal{F}_{r}$ has at most $\left(\begin{array}{l}n-1 \\ r-1\end{array}\right)$ members, and this bound is achieved if and only if $\mathcal{F}_{r}$ is a star. Hence, $|\mathcal{F}| \leq \sum_{r=1}^{u}\left(\begin{array}{l}n-1 \\ r-1\end{array}\right)$, and this bound is achieved if and only if $\mathcal{F}$ is the union of stars $\mathcal{F}_{1}, \ldots, \mathcal{F}_{u}$ whose members, since $\mathcal{F}$ is intersecting, must each contain some fixed element, namely that in the single member of $\mathcal{F}_{1}$.

Considering the results of Theorem 25 leads naturally to the following question.

Question 26. For each given value $u$, must each $(2, s)$-conditionally intersecting family $\mathcal{F} \subseteq$ $\left(\begin{array}{c}{[n]} \\ \leq u\end{array}\right)$ of maximal size for such families necessarily be of one of the forms (12)-(14)? 
In general, this appears to be a difficult question. We can however answer it positively for sufficiently large values $n$ with the next - and last - result of this paper, Theorem 27.

Theorem 27. Let $\mathcal{F} \subseteq\left(\begin{array}{c}{[n]} \\ \leq u\end{array}\right)$ be $(2, s)$-conditionally intersecting for $\frac{s}{2}<u<s-1$. If $s=2 k$, then

$$
|\mathcal{F}| \leq\left(\begin{array}{l}
n-1 \\
k-1
\end{array}\right)+\sum_{i=k+1}^{u}\left(\begin{array}{l}
n \\
i
\end{array}\right),
$$

and, for $n$ sufficiently larger than $s-r$, equality holds if and only if, up to isomorphism,

$$
\mathcal{F}=\left\{F \in\left(\begin{array}{c}
{[n]} \\
k
\end{array}\right): 1 \in F\right\} \cup\left\{F \in 2^{[n]}: k<|F| \leq u\right\} .
$$

If $s=2 k-1$, then

$$
|\mathcal{F}| \leq \sum_{i=k}^{u}\left(\begin{array}{l}
n \\
i
\end{array}\right)
$$

and, for $n$ sufficiently larger than $s-r$, equality holds if and only if, up to isomorphism,

$$
\mathcal{F}=\left\{F \in 2^{[n]}: k \leq|F| \leq u\right\} .
$$

Proof. As in the proof of Theorem 23, the bounds of Theorem 27 hold when $\mathcal{F}$ contains no set of size less than $k=\left\lceil\frac{s}{2}\right\rceil$, as do the characterisations of the extremal families.

Suppose then that $\mathcal{F}_{r} \neq \emptyset$ for some positive integer $r<k$. To conclude the proof, we will prove that $\mathcal{F}$ cannot achieve the bounds of the theorem for $n$ sufficiently larger than $s$. By adding sets to $\mathcal{F}$, we may suppose that $\mathcal{F}$ is maximal among $(2, s)$-conditionally intersecting subfamilies of $\left(\begin{array}{c}{[n]} \\ \leq u\end{array}\right)$ that contain some member of size strictly less than $k$. Define

$$
\mathcal{F}^{\prime}=\left(\mathcal{F} \cup \bigcup_{r=s-u}^{k-1} \sigma_{s-r}\left(\left(\mathcal{F}_{r}\right)^{C}\right)\right)-\bigcup_{r=1}^{k-1} \mathcal{F}_{r} .
$$

By the proof of Theorem 23, $\mathcal{F}^{\prime}$ is $(2, s)$-conditionally intersecting. Since $\mathcal{F}^{\prime}$ contains no set of size smaller than $k$ or any set larger than $u$, the first part of the proof shows that $\left|\mathcal{F}^{\prime}\right|$ satisfies the bounds of the theorem. We will prove that $|\mathcal{F}|<\left|\mathcal{F}^{\prime}\right|$ for $n$ sufficiently larger than $s$, implying that $|\mathcal{F}|$ cannot achieve the given bounds.

As in the proof of Theorem 23, $\mathcal{F} \cap \sigma_{s-r}\left(\left(\mathcal{F}_{r}\right)^{C}\right)=\emptyset$ for each $r$, so

$$
\left|\mathcal{F}^{\prime}\right|=|\mathcal{F}|+\sum_{r=s-u}^{k-1}\left|\sigma_{s-r}\left(\left(\mathcal{F}_{r}\right)^{C}\right)\right|-\sum_{r=1}^{k-1}\left|\mathcal{F}_{r}\right| .
$$

Thus to prove that $|\mathcal{F}|<\left|\mathcal{F}^{\prime}\right|$ for $n$ sufficiently larger than $s-r$, we must prove that

$$
\sum_{r=r^{\prime}}^{k-1}\left|\mathcal{F}_{r}\right|<\sum_{r=s-u}^{k-1}\left|\sigma_{s-r}\left(\left(\mathcal{F}_{r}\right)^{C}\right)\right|
$$


for $n$ sufficiently larger than $s-r$, where $r^{\prime}$ is the smallest integer for which $\mathcal{F}_{r^{\prime}} \neq \emptyset$. By the proof of Theorem 23. (15) is true when $r^{\prime} \geq s-u$, so suppose that $r^{\prime}<s-u(\leq k)$. For each integer $r$ with $s-u \leq r \leq k-1$, Theorem 24 implies that

$$
\frac{\left(\begin{array}{c}
n \\
s-r
\end{array}\right)}{\left(\begin{array}{c}
n \\
r
\end{array}\right)}\left|\mathcal{F}_{r}\right| \leq\left|\sigma_{s-r}\left(\left(\mathcal{F}_{r}\right)^{C}\right)\right| .
$$

Furthermore for such $r$, we have $r \leq k-1 \leq(2 k-1)-k=s-k<s-r$, so $\left(\begin{array}{c}n \\ s-r\end{array}\right) /\left(\begin{array}{c}n \\ r\end{array}\right) \rightarrow \infty$ for $n-(s-r) \rightarrow \infty$.

For each $r=s-u\left(>r^{\prime}\right), \ldots, k-1$ and $r^{\prime \prime}=r^{\prime}, \ldots, s-u-1$, consider any $A \in \mathcal{F}_{r^{\prime \prime}}$ and $B \supset A$ with $|B|=r$. If $B \notin \mathcal{F}_{r}$, then adding $B$ to $\mathcal{F}$ would create a larger $(2, s)$-conditionally intersecting subfamily of $\left(\begin{array}{c}{[n]} \\ \leq u\end{array}\right)$ with at least one member of size less than $k$, contradicting the maximality of $\mathcal{F}$. Hence, $B \in \mathcal{F}_{r}$. Double counting the pairs $(A, B)$ with $A \in \mathcal{F}_{r^{\prime \prime}}$ and $A \subset B \in \mathcal{F}_{r}$ yields the inequality

$$
\left|\mathcal{F}_{r}\right|\left(\begin{array}{c}
r \\
r^{\prime \prime}
\end{array}\right) \geq\left(\begin{array}{c}
n-r^{\prime \prime} \\
r-r^{\prime \prime}
\end{array}\right)\left|\mathcal{F}_{r^{\prime \prime}}\right|
$$

In particular, there is a constant $C$ such that $\sum_{r=r^{\prime}}^{s-u-1}\left|\mathcal{F}_{r}\right|<C\left|\mathcal{F}_{k-1}\right|$ for sufficiently large $n$. Hence, for $n$ sufficiently larger than $s-r$,

$$
\sum_{r=r^{\prime}}^{k-1}\left|\mathcal{F}_{r}\right|<\sum_{r=s-u}^{k-1} \frac{\left(\begin{array}{c}
n \\
s-r
\end{array}\right)}{\left(\begin{array}{c}
n \\
r
\end{array}\right)}\left|\mathcal{F}_{r}\right| \leq \sum_{r=s-u}^{k-1}\left|\sigma_{s-r}\left(\left(\mathcal{F}_{r}\right)^{C}\right)\right|,
$$

and thus $|\mathcal{F}|<\left|\mathcal{F}^{\prime}\right|$. This concludes the proof.

\section{Acknowledgements}

We thank the referees for their corrections and suggestions which improved the presentation of this paper.

\section{References}

[1] Rudolf Ahlswede and Levon H. Khachatrian. The complete nontrivial-intersection theorem for systems of finite sets. J. Combin. Theory Ser. A, 76(1):121-138, 1996.

[2] Rudolf Ahlswede and Levon H. Khachatrian. The complete intersection theorem for systems of finite sets. European J. Combin., 18(2):125-136, 1997.

[3] A. E. Brouwer, C. F. Mills, W. H. Mills, and A. Verbeek. Counting families of mutually intesecting sets. Electron. J. Combin., 20(2):Paper 8, 8, 2013.

[4] William Y. C. Chen, Jiuqiang Liu, and Larry X. W. Wang. Families of sets with intersecting clusters. SIAM J. Discrete Math., 23(3):1249-1260, 2009. 
[5] P. Erdős, Chao Ko, and R. Rado. Intersection theorems for systems of finite sets. Quart. J. Math. Oxford Ser. (2), 12:313-320, 1961.

[6] P. Frankl. On Sperner families satisfying an additional condition. J. Combinatorial Theory Ser. A, 20(1):1-11, 1976.

[7] P. Frankl. Shadows and shifting. Graphs Combin., 7(1):23-29, 1991.

[8] P. Frankl and Z. Füredi. Nontrivial intersecting families. J. Combin. Theory Ser. A, 41(1):150-153, 1986.

[9] Peter Frankl. The shifting technique in extremal set theory. In Surveys in combinatorics 1987 (New Cross, 1987), volume 123 of London Math. Soc. Lecture Note Ser., pages 81-110. Cambridge Univ. Press, Cambridge, 1987.

[10] Peter Frankl and Zoltán Füredi. A new generalization of the Erdős-Ko-Rado theorem. Combinatorica, 3(3-4):341-349, 1983.

[11] Zoltán Füredi and Lale Özkahya. Unavoidable subhypergraphs: a-clusters. In European Conference on Combinatorics, Graph Theory and Applications (EuroComb 2009), volume 34 of Electron. Notes Discrete Math., pages 63-67. Elsevier Sci. B. V., Amsterdam, 2009 .

[12] A. J. W. Hilton and E. C. Milner. Some intersection theorems for systems of finite sets. Quart. J. Math. Oxford Ser. (2), 18:369-384, 1967.

[13] Gy. Katona. Intersection theorems for systems of finite sets. Acta Math. Acad. Sci. Hungar, 15:329-337, 1964.

[14] Peter Keevash and Dhruv Mubayi. Set systems without a simplex or a cluster. Combinatorica, 30(2):175-200, 2010.

[15] Noam Lifshitz. On set systems without a simplex-cluster and the Junta method. arXiv preprint arXiv:1804.01026, 2018.

[16] Dhruv Mubayi. Erdős-Ko-Rado for three sets. J. Combin. Theory Ser. A, 113(3):547-550, 2006.

[17] Dhruv Mubayi. An intersection theorem for four sets. Adv. Math., 215(2):601-615, 2007.

[18] Dhruv Mubayi and Reshma Ramadurai. Set systems with union and intersection constraints. J. Combin. Theory Ser. B, 99(3):639-642, 2009.

[19] Dhruv Mubayi and Reshma Ramadurai. Simplex stability. Combin. Probab. Comput., 18(3):441-454, 2009. 